\title{
Intranasal injection of recombinant human erythropoietin improves cognitive and visual impairments in chronic cerebral ischemia rats
}

\author{
YANHUI ZHOU ${ }^{1}$, BIN SUN ${ }^{2}$, JUNHONG GUO ${ }^{3}$ and GUOHONG ZHOU ${ }^{4}$ \\ Departments of ${ }^{1}$ Internal Medicine and ${ }^{2}$ Orbitopathy, Shanxi Eye Hospital, Taiyuan, Shanxi 030002; \\ ${ }^{3}$ Department of Neurology, The First Hospital of Shanxi Medical University, Taiyuan, Shanxi 030001; \\ ${ }^{4}$ Department of Lacrimal Duct, Shanxi Eye Hospital, Taiyuan, Shanxi 030002, P.R. China
}

Received December 24, 2019; Accepted July 13, 2020

DOI: $10.3892 /$ br.2020.1347

\begin{abstract}
The present study aimed to study the protective effect of intranasally delivered recombinant human erythropoietin (rhEPO) on cognitive and visual impairments in a permanent bilateral common carotid artery occlusion (2VO)-induced chronic cerebral ischemia (CCI) rat model. Male Sprague-Dawley rats (age, 6 months) with 2VO-induced CCI were treated with intranasal $\mathrm{rhEPO}(50 \mathrm{U} / 100 \mathrm{~g})$ once per week for 8 weeks. A Morris water maze was used to evaluate the spatial learning and memory of the rats. Flash visual evoked potentials were measured to assess retinal function. Hematoxylin and eosin staining was performed to visualize and evaluate histopathological changes in the cerebral cortex, the hippocampus CA1 region and the retina. CCI-induced learning, memory and visual impairments were significantly alleviated in rats treated with rhEPO compared with those treated with a saline vehicle control. This was evidenced by remarkably decreased escape latency, increased frequency of crossing the hidden platform and elevated amplitude of primary wave in the rats treated with rhEPO. In addition, the rats experienced CCI-induced histopathological alterations, demonstrated by thinning of the cerebral cortex and retina, and losses of neurons and retinal ganglion cells. These alterations were significantly reversed in response to rhEPO administration compared with the saline vehicle control group. rhEPO may exert a protective role against cognitive and visual impairments in rats with $\mathrm{CCI}$ at least partially through preventing the thinning of the cerebral cortex and retina, as well as by inhibiting the loss of neurons and retinal ganglion cells.
\end{abstract}

Correspondence to: Dr Bin Sun, Department of Orbitopathy, Shanxi Eye Hospital, 100 Fudong, Taiyuan, Shanxi 030002, P.R. China

E-mail: sunbineye@163.com

Key words: erythropoietin, chronic cerebral hypoperfusion, bilateral common carotid artery occlusion, flash visual evoked potentials

\section{Introduction}

Erythropoietin (EPO) is a single chain glycoprotein that promotes hematopoietic cell growth through binding to the EPO receptor; recombinant human EPO (rhEPO) has previously been successfully used in the treatment of severe anemia (1-3). EPO is primarily produced in the kidneys but is also detectable in other organs, such as the liver and the brain (4). In addition to hematopoietic growth-promoting activity, EPO has been shown to exhibit anti-apoptotic and neuroprotective effects in acute hypoxic/ischemia cerebral damages (5). However, the effect of EPO treatment on chronic cerebral ischemia (CCI) remains relatively unknown.

A major challenge for clinical application of EPO in cerebral ischemia is to facilitate its delivery to the central nervous system (CNS) across the blood-brain barrier (BBB) (6). Current approaches for EPO delivery to the CNS in animal models include systemic [intraperitoneal (i.p.) or subcutaneous], intracerebroventricular (i.c.v.) and intranasal administrations. Because only $0.5-1 \%$ of systemically administered EPO can cross the BBB (7), higher doses of EPO are required to reach effective concentrations in the brain, leading to severe side effects such as cerebral infarct and overproduction of platelets (8-11). Although direct i.c.v. injection of EPO has been shown to provide significant protection against ischemic brain injury (12-14), it may not be suitable for clinical use owing to high risks of brain damage and infections (7). Compared with systemic and i.c.v. administrations, the intranasal delivery method has significant clinical potential owing to simple and non-invasive drug administration, rapid CNS delivery and minimal systemic exposure (15). A previous study showed that the brain uptake of neurotherapeutics after intranasal delivery is $>5$ times the uptake after i.p. delivery (16). The present study established a rat model of permanent bilateral common carotid artery occlusion (2VO)-induced CCI that were treated with intranasal rhEPO in order to explore the protective effects of rhEPO on cognitive and visual impairments. Morris water maze (MWM) and flash visual evoked potential (FVEP) measurement were used to evaluate learning and memory abilities, and visual functions in rats, respectively. Hematoxylin and eosin (H\&E) staining was performed to examine the histopathologic changes in the brain and retina. 


\section{Materials and methods}

Animals. Healthy male Sprague-Dawley rats ( $\mathrm{n}=90$; age, 6 months; weight, 300-340 g) were purchased from the Central Animal House at Shanxi Medical University and maintained under standard conditions according to the Animal Research Center guidelines of Shanxi Medical University (Taiyuan, China). Rats were acclimatized for 1 week before use. All rats were housed in rooms kept at $22^{\circ} \mathrm{C}$ with $60 \%$ humidity, and a 12-h light/dark cycle. Rats were provided ad libitum access to food and water. All the experimental procedures were approved by the Animal Use and Care Committee of Shanxi Medical University. All animal experiments were performed in accordance with the guidelines for Animal Use and Care of Shanxi Medical University (Taiyuan, China).

$2 \mathrm{VO}$ procedure. After a qualifying $\mathrm{MWM}$ test, rats were randomly divided into sham-operated, $2 \mathrm{VO}+$ saline and $2 \mathrm{VO}+$ rhEPO groups ( $\mathrm{n}=30 \mathrm{rats} / \mathrm{group}$ ). The $2 \mathrm{VO}$ surgery was performed to induce CCI as previously described (17). Briefly, rats were fasted for $12 \mathrm{~h}$ and deprived of water for $4 \mathrm{~h}$ followed by an i.p. injection of $5 \%$ (mass/volume) chloral hydrate $(325 \mathrm{mg} / \mathrm{kg}$, the dose for anesthesia before model establishment, Wuhan HeChang Chemical Co., Ltd.). The rats were immobilized, shaved and disinfected. The skin and mucosa at the front of the neck were cut and the muscles were bluntly separated to expose the bilateral common carotid arteries and the vagus nerve. The bilateral common carotid arteries were tightly double ligated with silk braided non-sutures (diameter, $26 \mathrm{~mm}$; model number, 3-0; Johnson \& Johnson). The wound was then rinsed thoroughly with metronidazole (Shijiazhuang No. 4 Pharmaceutical Co., Ltd.) and sutured layer by layer. After recovery, rats were returned to their home cages with free access to food and water. Rats in the sham-operated group were subjected to the same procedure without ligation of the common carotid arteries (17). The body weight loss was $<3 \%$ post-fasting for $12 \mathrm{~h}$.

Intranasal drug delivery. rhEPO (Shenyang Sunshine Pharmaceutical Co., Ltd.; 3Sbio Group) was dissolved in sterile saline (Shijiazhuang No. 4 Pharmaceutical Co., Ltd.) at a concentration of $150 \mathrm{U}$ rhEPO/120 $\mu \mathrm{l}$. At 3 days following the $2 \mathrm{VO}$ procedure, $2 \mathrm{VO}+$ rhEPO group was intranasally administered with rhEPO (50 U/100 g/week; Shenyang Sunshine Pharmaceuticals Co., Ltd.; 3Sbio Group) for 8 weeks. Sham-operated and $2 \mathrm{VO}+$ saline groups were given an equivalent volume of saline. Briefly, rats were anesthetized with 5\% (mass/volume) chloral hydrate $(325 \mathrm{mg} / \mathrm{kg})$ by i.p. injection followed by stereotaxic frame (Chengdu Taimeng Technology \& Market Co. Ltd.,)-based immobilization in a supine position at a $-70^{\circ}$ angle (18). A polyvinyl chloride tube (inner diameter: $0.5 \mathrm{~mm}$, outer diameter: $1.0 \mathrm{~mm}$; Jiangxi Hongda Medical Equipment Group Co., Ltd.) attached to a $1 \mathrm{ml}$ syringe (Jiangxi Hongda Medical Equipment Group Co., Ltd.) was inserted into each nostril to a depth of $\sim 2 \mathrm{~cm}$. A total of $125 \mu \mathrm{l}$ of $\mathrm{rhEPO}$ sterile solution containing $150 \mathrm{U}$ rhEPO in $120 \mu \mathrm{l}$ of saline was delivered by gently pushing into the nasal cavities for $30 \mathrm{~min}$. After delivery, the tube was removed, and the rats were kept immobilized for $30 \mathrm{~min}$ to avoid the loss of rhEPO. Rats that swallowed or expelled fluid were excluded from further analyses. A total of eight rats in each group were included in the statistical analysis. The other 12 rats were excluded as they did not meet the test standards for various reasons.

MWM test. Rats were subjected to MWM test for spatial learning and memory evaluation before 2VO surgery and after 8 weeks of rhEPO/saline treatment, respectively. A black circular pool (diameter, $150 \mathrm{~cm}$; height, $50 \mathrm{~cm}$ ) divided into four equal quadrants and with four points designed as starting positions (I, II, III and IV) was filled with water at $24^{\circ} \mathrm{C}$. A video camera was hanging over the pool, and a black platform (diameter, $10 \mathrm{~cm}$; height, $27 \mathrm{~cm}$ ) was submerged $2 \mathrm{~cm}$ below the surface of the water. The position of each reference inside and outside the maze was kept unchanged during the training sessions (19).

Before 2VO surgery, each rat received one training session per day for 5 days consecutively $(19,20)$. Each session consisted of four trials with an interval of $1 \mathrm{~h}$. During each trial, the rat was gently placed in the water at one of the four starting positions and faced toward the wall of the maze. The time spent to find the hidden platform (escape latency) and the swimming trajectories were recorded. If a rat failed to find the platform within $2 \mathrm{~min}$, the rat would be gently guided to the platform and was allowed to stay on it for $5 \mathrm{sec}$ before being removed from the water. A maximum score of 2 min was assigned. On day six, the platform was removed, and the rats were subjected to a probe test for spatial memory assessment. Another probe test was performed after 8 weeks of rhEPO or saline treatment. The number of times a rat passed through the target platform within 2 min was recorded.

FVEP measurement. FVEP, a cluster of electrical signals that occur in the cerebral cortex in response to visual stimuli (21), was determined for visual function evaluation. As two rats did not cooperate, in order to unify the several indices in the experiments, a total of six rats successfully administered drugs were selected from each group and were anesthetized with chloral hydrate, such that comparisons could be performed using paired statistics. Latency and amplitude of the primary wave (P1) were recorded using a four channel signal averager (Nicolet Biomedical Inc.).

$H \& E$ staining. After MWM test and FVEP measurement, rats were anesthetized with $5 \%$ chloral hydrate and sacrificed by intracardial perfusion with $0.9 \%$ saline $(200-240 \mathrm{ml}$; Shijiazhuang No.4 pharmaceutical Co. Ltd.,) followed by $4 \%$ paraformaldehyde (250-400 ml; Tianjin Aoran Fine Chemical Research Institution) in $0.1 \mathrm{~mol} / \mathrm{l}$ PBS (pH 7.4; Tianjin Aoran Fine Chemical Research Institution). When there is no sign of breath, the rats were deemed dead. The brain and eyes of each rat were removed and fixed in $10 \%$ formaldehyde for $48 \mathrm{~h}$ followed by dehydration in a graded series of ethanol and paraffin embedding. Cerebral cortex, hippocampus CA1 and retina tissues were cut into sections $(5 \mu \mathrm{m})$. The paraffin-embedded sections were dewaxed in xylene, dehydrated using a series of graded ethanol solutions ( 2 min each in 100, 90 and 70\%), washed in distilled water, stained with H\&E (Chengdu Kelong Chemical Reagent Factory), dehydrated through graded ethanol solutions (95\% ethanol $15 \mathrm{sec}, 100 \%$ ethanol $10 \mathrm{~min}$ ), soaked for $10 \mathrm{~min}$ in turn in $100 \%$ xylene (I,II Tianjin Damao Chemical Reagent Factory) and mounted using neutral balsam (Sinopharm Chemical Reagent Co., Ltd.). 

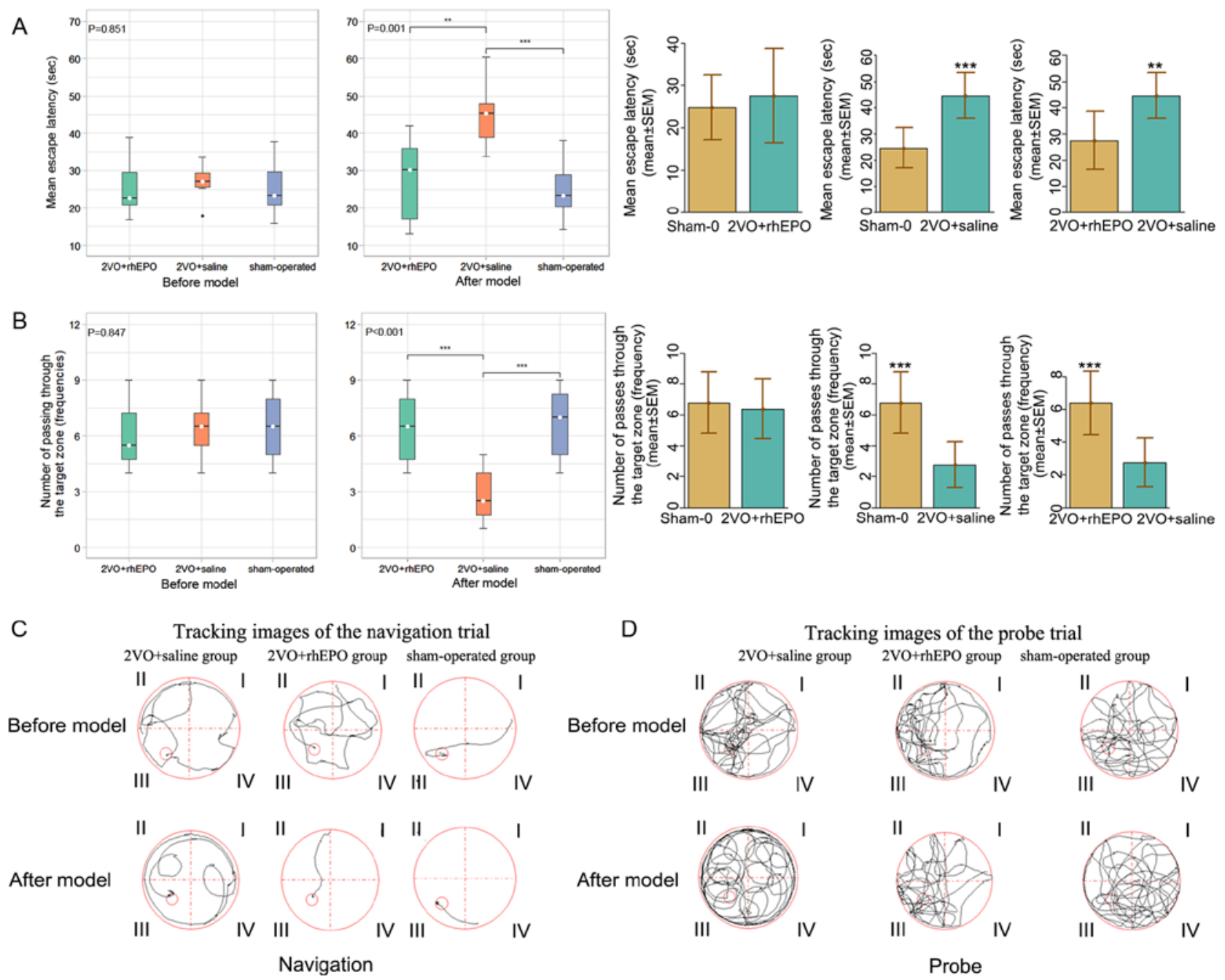

Figure 1. MWM test. MWM test was performed before the chronic cerebral ischemia model was established and after treatment with rhEPO. The (A) escape latency and (B) the number of passes through the target zone were recorded. Left, two-way ANOVA was performed, followed by a LSD multiple comparisons test. Right, differences between groups were compared using a one-way ANOVA and a LSD-t test. $\mathrm{n}=8$ rats/group. ${ }^{* *} \mathrm{P}<0.01$, ${ }^{* * *} \mathrm{P}<0.001$. Tracking images of (C) the navigation trial and (D) the probe trial. MWM, Morris water maze; 2VO, bilateral common carotid artery occlusion; rhEPO, recombinant human erythropoietin.

Morphological changes were observed using an optical microscopy (Chengdu Taimeng Technology \& Market Co., Ltd.). The thicknesses of the cerebral cortex and the retina as well as the number of hippocampus CA1 neurons and retinal ganglion cells were measured using a light microscope (Olympus BX60F5; Olympus Corporation) and a BI-2000 medical image analysis system (Chengdu Taimeng Technology \& Market Co., Ltd.).

Statistical analysis. Data were expressed as mean \pm the standard error of the mean. Statistical analysis was performed using R (22) version 3.5.1 and R-Studio version 1.0.44 (23). Differences between groups were compared using a one-way ANOVA with a post-hoc LSD t-test, or a two-way ANOVA, followed by a LSD multiple comparisons test. $\mathrm{P}<0.05$ was considered to indicate a statistically significant difference.

\section{Results}

\section{Clinical observation}

Model establishment and behavioral observation after surgery. The rats were awake $\sim 1 \mathrm{~h}$ after surgery. The main clinical symptoms included drowsiness, poor spirit, apathy, a lack of activity, limbs failing to fully stretch, vacillation to the left and to the right when walking and a proneness to falling.

Rats after saline or rhEPO treatment. After treatment, the control group (2VO + saline) rats were less active, slow-moving, had memory deficits and exhibited a lower sense of pain. One of the rats in the control group repeatedly bit other rats. Rats in the experimental group $(2 \mathrm{VO}+\mathrm{rhEPO})$ and the sham-operated group were much more active and more mentally quick and agile compared with the rats in the control group.

rhEPO protects CCI model rats from learning and memory impairments. To investigate the effect of rhEPO on learning and memory impairments in CCI, the present study established a 2VO-induced CCI rat model and treated the rats with rhEPO once a week for 8 weeks. MWM training and tests were performed to evaluate spatial learning and memory in rats before modeling and 8 weeks after modeling/treatment. As shown in Fig. 1A (left), no significant difference was observed in the escape latency among the three groups $(\mathrm{P}=0.851)$ before 

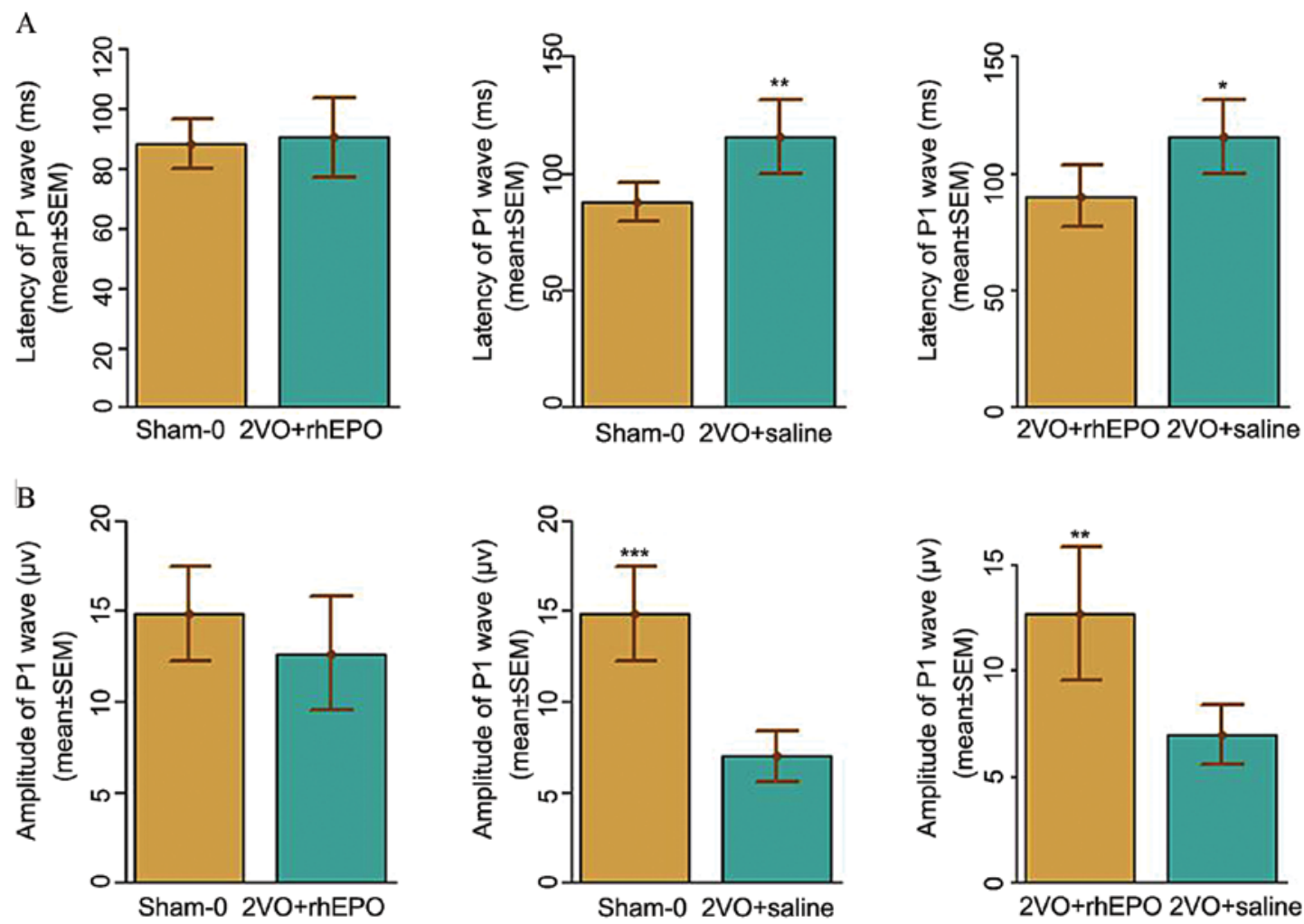

Figure 2. Flash visual evoked potential measurement. (A) Latency and (B) amplitude of the $\mathrm{P} 1$ wave. $\mathrm{n}=6$ rats/group. ${ }^{*} \mathrm{P}<0.05,{ }^{* * *} \mathrm{P}<0.01,{ }^{* * *} \mathrm{P}<0.001$. $2 \mathrm{VO}$, bilateral common carotid artery occlusion; rhEPO, recombinant human erythropoietin; P1, primary wave; Sham- 0 , sham-operated.

modeling. After modeling, there was a significant difference in the escape latency between the three groups $(\mathrm{P}=0.000)$. After 8 weeks of modeling and treatment, as shown in Fig. 1A (right) the escape latency in the $2 \mathrm{VO}+$ saline group was significantly increased compared with that in the sham-operated group $(\mathrm{P}=0.000)$, which indicated that there was an impaired spatial learning ability in the saline-treated $\mathrm{CCI}$ rats. By contrast, the escape latency in the experimental group $(2 \mathrm{VO}+$ rhEPO) was significantly lower compared with that in the control group $(2 \mathrm{VO}+$ saline $)(\mathrm{P}=0.001)$ and remained comparable with the sham-operated group $(\mathrm{P}=0.549)$.

In addition, the frequencies of passing through the target zone were comparable among the three groups before modeling $(\mathrm{P}=0.847$; Fig. 1B, left), whereas after modeling, there was a significant difference in the number of passes through the target zone between the three groups $(\mathrm{P}=0.000)$. After 8 weeks of modeling and treatment, as shown in Fig. 1B (right), the rats in the $2 \mathrm{VO}+$ saline treatment group crossed the target zone significantly less often than those in the sham-operated group after the model/treatment was established $(\mathrm{P}=0.000)$, which suggested that there may be a memory deficit in rats with 2VO-induced CCI. By contrast, the frequency of passing through the target zone in rhEPO-treated CCI rats was significantly higher compared with that in saline-treated ones $(\mathrm{P}=0.001)$ and remained comparable with the sham-operated group $(\mathrm{P}=0.591)$, which suggested that there was a protective role of rhEPO against memory impairment in CCI rats. Similar results were observed in the swimming trajectory length (Fig. 1C and D). Taken together, these data suggested that rhEPO may provide effective protection against learning and memory deficits in rats with CCI.
rhEPO improves visual dysfunction in rats with CCI. As cerebral ischemia has previously been shown to cause retinal damage, which can lead to visual function impairment and subsequent deteriorated spatial learning ability $(24,25)$, the present study next sought to investigate the effect of rhEPO on visual functions in CCI model rats. As shown in Fig. 2, the results of FVEP measurements indicated that the latency and amplitude of the P1 wave were significantly increased and decreased, respectively, in the $2 \mathrm{VO}+$ saline group compared with those in the sham-operated group, which suggested that $\mathrm{CCI}$ induced visual dysfunction in rats with 2VO. Importantly, rhEPO treatment markedly reduced the latency and elevated the amplitude of the P1 wave compared with the $2 \mathrm{VO}+$ saline treatment group, which suggested that rhEPO may restore some visual function in CCI rats, which in turn improves the spatial learning ability in these rats.

rhEPO reverses neuropathological and retinal alterations in rats with CCI. To further study the histopathological basis of the improved learning and memory impairments in rhEPO-treated rats, $H \& E$ staining was performed to observe the morphological changes in the hippocampal CA1 region, which is known to be highly involved in learning and memory (26), as well as morphological changes in the retina in response to rhEPO administration. Neurons with abnormal nuclear shape and massive vacuolation of cytoplasm were loosely and irregularly arranged in the control $(2 \mathrm{VO}+$ saline $)$ group. By contrast, in the experimental $(2 \mathrm{VO}+\mathrm{rhEPO})$ group, neuronal cells with normal size and morphology were tightly packed and ordered. In addition, the thickness of cerebral cortex was significantly decreased in the $2 \mathrm{VO}+$ saline group compared with that in the 

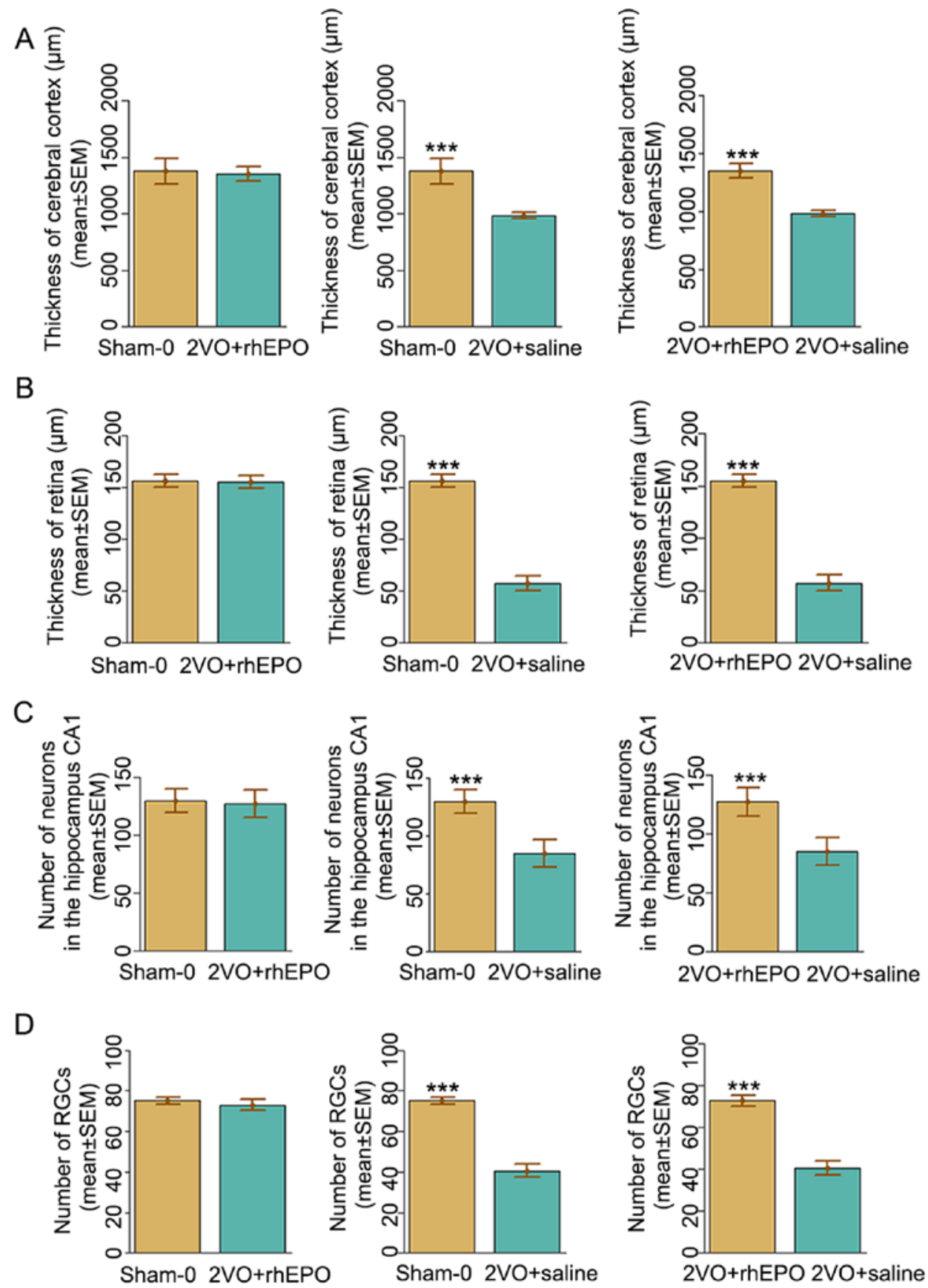

Figure 3. Quantification of the hematoxylin and eosin stained tissues. The thicknesses of (A) cerebral cortex and (B) retina as well as the (C) numbers of neurons in the hippocampus CA1 and (D) retinal ganglion cells were quantified from the images presented in Fig. 3. Data are expressed as the mean \pm SEM; $\mathrm{n}=6$ rats/group. ${ }^{* *} \mathrm{P}<0.001 .2 \mathrm{VO}$, bilateral common carotid artery occlusion; rhEPO, recombinant human erythropoietin.

sham-operated group (982 $\pm 28 \mu \mathrm{m}$ vs. $1378 \pm 113 \mu \mathrm{m})$, which was significantly improved in the $2 \mathrm{VO}+\operatorname{rhEPO}$ group $(1,350 \pm 63 \mu \mathrm{m}$ vs. $982 \pm 28 \mu \mathrm{m}, \mathrm{F}=49.645, \mathrm{P}<0.001$ ) (Fig. $3 \mathrm{~A}$ ). Similar results were observed in the thickness of retina (Fig. 3B), the number of neurons in hippocampus CA1 area (Fig. 3C) and the number of retinal ganglion cells (Fig. 3D). These data collectively suggested a protective role of rhEPO against neuronal and retinal damage in rats with $\mathrm{CCI}$.

\section{Discussion}

Intranasal delivery of neurotherapeutics is widely used in animal experiments owing to its simple and non-invasive administration, repeatability, and rapid and effective CNS delivery (15). EPO is a heat-resistant acidic glycoprotein containing sialic acid and is typically used to treat different types of anemia $(1,3)$. In addition, EPO has been shown to exert neuroprotective effects on acute ischemia in the CNS through various mechanism (27), such as inhibiting glutamate overproduction (28), inflammation $(29,30)$ and oxidative stress (31). It has been reported that intranasal administration of EPO is a non-invasive, simple approach to bypass the BBB (32) and is safer, 10-times more rapid and more efficient than intravenous delivery in acute brain injury animal models (33). However, the effect of intranasally delivered EPO on CCI-induced brain and retina damage remains unclear. The present study explored 
the effects of intranasally delivered rhEPO on behavior, spatial orientation, learning and memory and vision function in rats with 2VO-induced CCI.

$\mathrm{CCI}$ is considered both a neurological and cerebrovascular disease and is characterized by progressive cognitive and behavioral deterioration caused by long-term cerebral blood perfusion insufficiency (34). Permanent $2 \mathrm{VO}$ in rats can induce cognitive impairment and has been widely used to establish CCI models (17). CCI is closely associated with several cerebrovascular diseases, including cerebral arteriosclerosis, cerebral infarction, vascular dementia and Alzheimer's disease. With an increasingly aging population, CCI is becoming a major cause of disability and constitutes a large burden to healthcare systems (34).

The MWM test performed in the present study showed a significant increase in escape latency, swimming trajectory length and a decreased frequency of passing through the target quadrant in the saline-treated animals 8 weeks after the $2 \mathrm{VO}$ procedure compared with the sham-operated group, which suggested that a CCI model was successfully established in the present study. Furthermore, these cognitive impairments were significantly improved in response to rhEPO administration compared with the $2 \mathrm{VO}+$ saline treatment group, which suggested a protective role of rhEPO against neuronal damage in CCI. The hippocampus is an important region of the brain that governs spatial learning and memory (26). Hippocampal neuronal damage and thinning of the cerebral cortex in response to hypoxia are possible mechanisms underlying cognitive and abnormal dysfunction in CCI. In the present study, it was found that the neurons in the cortex and hippocampus CA1 region exhibited morphological changes and the overall number of neurons was significantly reduced in the $2 \mathrm{VO}+$ saline group compared with those observed in the sham group, which is consistent with previous studies (17). Importantly, these pathological alterations were noticeably reversed in the rhEPO-treated rats compared with the saline-treated ones, further confirming that rhEPO may alleviate neuronal damage in CCI.

Ocular ischemic syndrome commonly occurs in the elderly, and is typically a local manifestation of internal diseases, such as high blood pressure, arteriosclerosis and diabetes (35). The ophthalmic artery is the first branch of the internal carotid artery (36). Thus, internal carotid artery occlusion may lead to retina ischemia. It has previously been reported that ocular ischemic syndrome is an early sign of cerebral ischemia and the occurrence between retinal and brain ischemia is significantly positively correlated (35). Retinal damage induces visual function impairment, which contributes to deteriorated spatial learning ability (24,25). Indeed, the present results showed that the latency and amplitude of the P1 wave were significantly increased and decreased, respectively, in the saline-treated CCI rats compared with those in the sham-operated group, which suggests that CCI may induce visual dysfunction in rats. Intriguingly, rhEPO treatment restored some visual function in CCI rats as evidenced by significantly reduced latency and elevated amplitude of P1 wave compared with $2 \mathrm{VO}+$ saline, which at least partially contributed to improvement of spatial learning ability in these rats. Consistent results were observed in the changes in the thickness of retina and the number of retinal ganglion cells. These data collectively suggested a potential protective role of rhEPO against retinal damage in rats with CCI.
Results from the present study suggested that intranasally delivered rhEPO may attenuate cognitive and visual impairments in 2VO-induced CCI in rats possibly through protecting the cerebral cortex and retina from thinning, and preventing losses of neurons and retinal ganglion cells. These data indicated that intranasal administration of rhEPO may be a potential therapeutic approach in CCI treatment. Further studies are required to reveal the molecular mechanism of rhEPO in CCI therapy.

\section{Acknowledgements}

Not applicable.

\section{Funding}

No funding was received.

\section{Availability of data and materials}

All data generated or analyzed during this study are included in this published article.

\section{Authors' contributions}

YZ and BS conceived and supervised the study, YZ designed and performed the experiments. JG made substantial contributions to the conception of the study and provided new tools and reagents. GZ developed new software and performed the simulations. YZ analyzed data and wrote the manuscript. YZ, BS and JG revised the manuscript. All authors reviewed the results and approved the final manuscript.

\section{Ethics approval and consent to participate}

All the experimental procedures were approved by the Animal Use and Care Committee in Shanxi Medical University (Taiyuan, China). All animal experiments were performed in accordance with the guidelines for Animal Use and Care of Shanxi Medical University (Taiyuan, China).

\section{Patient consent for publication}

Not applicable.

\section{Competing interests}

The authors declare that they have no competing interests.

\section{References}

1. Miller JL, Church TJ, Leonoudakis D, Lariosa-Willingham K, Frigon NL, Tettenborn CS, Spencer JR and Punnonen J: Discovery and characterization of nonpeptidyl agonists of the tissue-protective erythropoietin receptor. Mol Pharmacol 88: 357-367, 2015.

2. Rainville N, Jachimowicz E and Wojchowski DM: Targeting EPO and EPO receptor pathways in anemia and dysregulated erythropoiesis. Expert Opin Ther Targets 20: 287-301, 2016.

3. Miller JL, Rai M, Frigon NL, Pandolfo M, Punnonen J and Spencer JR: Erythropoietin and small molecule agonists of the tissue-protective erythropoietin receptor increase FXN expression in neuronal cells in vitro and in Fxn-deficient KIKO mice in vivo. Neuropharmacology 123: 34-45, 2017. 
4. Wang L, Wang X, Su H Han Z, Yu H, Wang D, Jiang R, Liu Z and Zhang J: Recombinant human erythropoietin improves the neurofunctional recovery of rats following traumatic brain injury via an increase in circulating endothelial progenitor cells. Transl Stroke Res 6: 50-59, 2015.

5. Ren Q, Zhang XF and Yang JY: Erythropoietin reduces white matter damage in two-day-old rats exposed to hypoxic/ischemia injury. Neurol Res 38: 1020-1026, 2016.

6. Zhang F, Xing J, Liou AK, Wang S, Gan Y, Luo Y, Ji X, Stetler RA, Chen J and Cao G: Enhanced delivery of erythropoietin across the blood-brain barrier for neuroprotection against ischemic neuronal injury. Transl Stroke Res 1: 113-121, 2010.

7. Brines $\mathrm{M}$ and Cerami A: Emerging biological roles for erythropoietin in the nervous system. Nat Rev Neurosci 6: 484-494, 2005.

8. Wolf RF, Peng J, Friese P, Gilmore LS, Burstein SA and Dale GL: Erythropoietin administration increases production and reactivity of platelets in dogs. Thromb Haemost 78: 1505-1509, 1997.

9. Wiessner C, Allegrini PR, Ekatodramis D, Jewell UR, Stallmach $\mathrm{T}$ and Gassmann M: Increased cerebral infarct volumes in polyglobulic mice overexpressing erythropoietin. J Cereb Blood Flow Metab 21: 857-864, 2001.

10. Leyland-Jones B, Investigators B and Study G: Breast cancer trial with erythropoietin terminated unexpectedly. Lancet Oncol 4 459-460, 2003.

11. Wun T, Law L, Harvey D, Sieracki B, Scudder SA and Ryu JK: Increased incidence of symptomatic venous thrombosis in patients with cervical carcinoma treated with concurrent chemotherapy, radiation, and erythropoietin. Cancer 98: 1514-1520, 2003.

12. Sadamoto Y, Igase K, Sakanaka M, Sato K, Otsuka H, Sakaki S, Masuda S and Sasaki R: Erythropoietin prevents place navigation disability and cortical infarction in rats with permanent occlusion of the middle cerebral artery. Biochem Biophys Res Commun 253: 26-32, 1998.

13. Sakanaka M, Wen TC, Matsuda S, Masuda S, Morishita E, Nagao $M$ and Sasaki R: In vivo evidence that erythropoietin protects neurons from ischemic damage. Proc Natl Acad Sci USA 95: 4635-4640, 1998 .

14. Bernaudin M, Marti HH, Roussel S, Divoux D, Nouvelot A, MacKenzie ET and Petit E: A potential role for erythropoietin in focal permanent cerebral ischemia in mice. J Cereb Blood Flow Metab 19: 643-651, 1999.

15. Alcalá-Barraza SR, Lee MS, Hanson LR, McDonald AA, Frey WH II and McLoon LK: Intranasal delivery of neurotrophic factors BDNF, CNTF, EPO, and NT-4 to the CNS. J Drug Target 18: 179-190, 2010.

16. Chauhan MB and Chauhan NB: Brain Uptake of neurotherapeutics after intranasal versus intraperitoneal delivery in Mice. J Neurol Neurosurg 2: 009, 2015.

17. Zhang T, Gu J, Wu L, Li N, Sun Y, Yu P, Wang Y, Zhang G and Zhang Z: Neuroprotective and axonal outgrowth-promoting effects of tetramethylpyrazine nitrone in chronic cerebral hypoperfusion rats and primary hippocampal neurons exposed to hypoxia. Neuropharmacology 118: 137-147, 2017.

18. Merelli A, Caltana L, Girimonti P, Ramos AJ, Lazarowski A and Brusco A: Recovery of motor spontaneous activity after intranasal delivery of human recombinant erythropoietin in a focal brain hypoxia model induced by $\mathrm{CoCl} 2$ in rats. Neurotox Res 20 : 182-192, 2011.

19. Patil SS, Sunyer B, Höger H and Lubec G: Evaluation of spatial memory of C57BL/6J and CD1 mice in the Barnes maze, the Multiple T-maze and in the Morris water maze. Behav Brain Res 198: 58-68, 2009.
20. Miskowiak KW, Vinberg M, Macoveanu J, Ehrenreich H, Køster N, Inkster B, Paulson OB, Kessing LV, Skimminge A and Siebner HR: Effects of erythropoietin on hippocampal volume and memory in mood disorders. Biol Psychiatry 78: 270-277, 2015.

21. Holmes MD and Sires BS: Flash visual evoked potentials predict visual outcome in traumatic optic neuropathy. Ophthalmic Plast Reconstr Surg 20: 342-346, 2004.

22. R Core Team. R: A language and environment for statistical computing. R Foundation for Statistical Computing, Vienna, Austria, 2012. ISBN 3-900051-07-0, URL http://www.R-project. org/.

23. R-Studio Team. R-Studio: Integrated Development for $\mathrm{R}$ RStudio, Inc., Boston, MA, 2015. URL http://www.rstudio.com/.

24. Brown RE and Wong AA: The influence of visual ability on learning and memory performance in 13 strains of mice. Learn Mem 14: 134-144, 2007

25. Kaur C, Foulds WS and Ling EA: Hypoxia-ischemia and retinal ganglion cell damage. Clin Ophthalmol 2: 879-889, 2008.

26. Bartsch T, Döhring J, Rohr A, Jansen O and Deuschl G: CA1 neurons in the human hippocampus are critical for autobiographical memory, mental time travel, and autonoetic consciousness. Proc Natl Acad Sci USA 108: 17562-17567, 2011.

27. Hu MC, Shi M, Cho HJ, Zhang J, Pavlenco A, Liu S, Sidhu S, Huang LJ and Moe OW: The erythropoietin receptor is a downstream effector of Klotho-induced cytoprotection. Kidney Int 84: 468-481, 2013.

28. Lourhmati A, Buniatian GH, Paul C, Verleysdonk S, Buecheler R, Buadze M, Proksch B, Schwab M, Gleiter CH and Danielyan L: Age-dependent astroglial vulnerability to hypoxia and glutamate: The role for erythropoietin. PLoS One 8: e77182, 2013.

29. Alnaeeli M, Raaka BM, Gavrilova O, Teng R, Chanturiya T and Noguchi CT: Erythropoietin signaling: A novel regulator of white adipose tissue inflammation during diet-induced obesity. Diabetes 63: 2415-2431, 2014

30. Vinberg M, Weikop P, Olsen NV, Kessing LV and Miskowiak K: Effect of recombinant erythropoietin on inflammatory markers in patients with affective disorders: A randomised controlled study. Brain Behav Immun 57: 53-57, 2016.

31. Ardalan MR, Estakhri R, Hajipour B, Ansarin K, Asl NA, Nasirizade MR, Azar AN, Ghorbanihaghjou A, Vatankhah AM and Esmaili HA: Erythropoietin ameliorates oxidative stress and tissue injury following renal ischemia/reperfusion in rat kidney and lung. Med Princ Pract 22: 70-74, 2013.

32. Yu YP, Xu QQ, Zhang Q, Zhang WP, Zhang LH and Wei EQ: Intranasal recombinant human erythropoietin protects rats against focal cerebral ischemia. Neurosci Lett 387: 5-10, 2005.

33. Fletcher L, Kohli S, Sprague SM, Scranton RA, Lipton SA, Parra A, Jimenez DF and Digicaylioglu M: Intranasal delivery of erythropoietin plus insulin-like growth factor-I for acute neuroprotection in stroke. Laboratory investigation. J Neurosurg 111: 164-170, 2009.

34. Zhu S, Min D, Zeng J, Ju Y, Liu Y and Chen X: Transplantation of Stem Cells from human exfoliated deciduous teeth decreases cognitive impairment from chronic cerebral ischemia by reducing neuronal apoptosis in rats. J Stem Cells Int 2020: 6393075, 2020.

35. Masuda T, Shimazawa M, Ishizuka F, Nakamura S, Tsuruma K and Hara $\mathrm{H}$ : Tissue kallikrein (kallidinogenase) protects against retinal ischemic damage in mice. Eur J Pharmacol 738: 74-82, 2014.

36. Bird B and Stawicki SP: Anatomy, head and neck, ophthalmic arteries. In: StatPearls. Treasure Island (FL), 2019. 